\title{
Study on Performance Analysis of HQAM for DCT and DWT Based Compressed Image Transmission over AWGN Channel
}

\author{
Rezaul Karim ${ }^{1}$, Shahela Pervin ${ }^{2}$, Umme Moon Ima ${ }^{2}$ and Md. Khaliluzzaman ${ }^{2}$ \\ ${ }^{1}$ Dept. of Computer Science \& Engineering, University of Chittagong (CU) \\ Chittagong-4331, Bangladesh \\ ${ }^{2}$ Dept. of Computer Science \& Engineering, International Islamic University Chittagong (IIUC) \\ Chittagong- 4203, Bangladesh
}

\begin{abstract}
Image transmission over a noisy channel is vital in the recent time because of reliable multimedia transmission. This topic has gained importance for the purpose of achieving less storage capacity and required bandwidth during transmitting the image through a noisy channel. In this paper, prior exertions of compressed image transmission are illustrated. These exertions are based on two compressed methods i.e., Discrete Wavelet transform (DWT) and Discrete Cosine Transform (DCT). Furthermore, overviews of the polar coding are presented which are used as encoder and decoder for converting the compressed quantized values to binary code streams. In addition, the overall advantages of the HQAM over the QAM modulation scheme are demonstrated in this paper. Finally, by exploring the preceding efforts, a method is proposed for future work. In the proposed method the performance of HQAM is analyzed for DWT and DCT after transmitting the image through the noisy AWGN channel.

Keywords - DCT, DWT, HQAM, AWGN, MATLAB, Fourier Transform
\end{abstract}

\section{Introduction}

Image data needs to be transmitted in most multimedia applications. Image compression is done before the transmission for efficient utilization of channel bandwidth. The processes of decreasing the size of the image without fallen down the quality of the image to unacceptable limit are image compression. The compressed images/videos require less memory to store and less time to transmit. The most widely used operation in image compression is Discrete cosine transform (DCT).To convert the input pixel values to frequency domain due to the computational efficiency of DCT different compression standards like JPEG, MPEG uses cosine function. Another efficient technique used for image compression is Discrete Wavelet Transform (DWT) based coding. Both DCT and DWT have the capacity to show the image at various exploration.Hybrid transform technique has been introduced by exploiting the convenience of both DCT and DWT. Here, propose the use of a modification of QAM technique i.e., Hierarchical Quadrature Amplitude Modulation (HQAM) technique for transmitting compressed images through AWGN channels. HQAM provides unequal error protection (UEP). This method without increasing the bandwidth various kind of protection for transmit data are achieved.

The paper is organized as follows. Discrete cosine transform and Discrete wavelet transform is described in Section II and III. The comparison of DCT and DWT is present in Section IV. Polar coding and conventional encoding are explained in Section V and VI. The explanation of HQAM is provided in Section VII. The comparison of QAM and HQAM is described in Section VIII. Performance analysis of AWGN channel is explained in Section IX. The proposed method is defined in Section X. In Section XI, the paper is concluded.

\section{Discrete Cosine Transform}

This section explains the Discrete Cosine Transform (DCT) procedures in details that are used in the different research works. Such as In [1], purposed a method of removing noise adorned in a speech signal represents the advantages of using the standard Discrete Fourier Transform (DFT) as compared to the Discrete Cosine Transform (DCT). Based on the statistical modeling of the DCT coefficients edification of the Minimum Mean Square Error (MMSE) filter is demonstrated. Also, it demonstrates based on the fact that speech energy edification of an over-attenuation factor is not always present in all coefficients or in the noisy signal at all times. The proposed methods in [1] were evaluated using both Gaussians distributed white noise as well as recorded fan noise against the noise reduction filter with favorable results. Although such as the Wavelet transform the algorithm is simulated with the DCT, it should also be applicable to other types of real transform.

In [2], exploration of compression using Discrete Cosine Transform is traced by choosing proper ambit method and for better PSNR result have been obtained. For image compression, the Joint Photographic Experts Group (JPEG) 
standard makes use of DCT. Discrete cosine transform (DCT) is for converting a signal into elementary frequency components and effort to de-correlate the data of an image. Independently each transform after de-correlation without losing compression efficiency coefficient can be encoded. The ratio between the highest possible strength of a signal and the ability to decompose in noise is PSNR and normally expressed in the logarithmic decibel scale and generally used as a degree of efficiency of reformation in compression of image etc. In this paper, they showed the process of DCT compression which is shown in Fig. 1. And Fig. 2 shows the decompressed image histogram of DCT decompressed image.

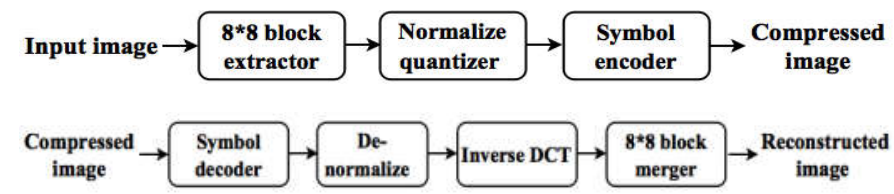

Fig. 1 Compression of imageby DCT [2].

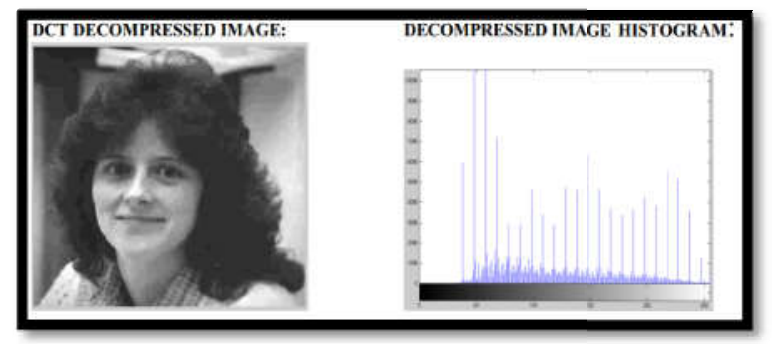

Fig. 2 Compression of image by DCT [2].

In [3], studied JPEG image compression algorithm which is used for full-color still image applications. And it describes all the components of it, lossy image compression using DCT. DCT is an orthogonal transformation used for compression data and maps an image space into a frequency. That has a static set of basis function file size with minimum image erosion by removing the least important information. The size is reduced by Joint Photographic Experts Group compression which is performed in sequential steps and to categorize compression techniques use two ways. Which is applied in images where we can wrinkle some of the best details in the image to protect a slender more bandwidth or storage space is Lossy compression. And at lossless Compression System without any distortion reducing the bit rate of the compressed image output is aimed. After decompression, the bit stream is uniform to the original bit stream. This paper also shows the compression step which is in Fig. 3(a) and decompression step is shown in Fig. 3(b).

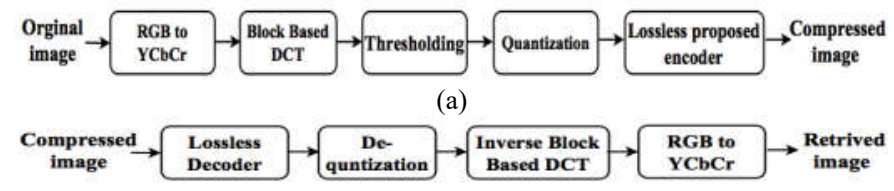

(b)

Fig. 3 Compression algorithm scheme: (a) compression step and (b) decompression step [3].

In [4], an efficient two-dimensional DCT operator is proposed for multimedia applications. One of the most extensively used operations is discrete cosine transform (DCT) in video/image compressions. This paper proposes SWP-based operator to speed up the computations process DCT too. Parallel computations are performed on multiple pixels packed in word size input registers by SWP-based DCT operator so that the performance of the operator is increased multimedia oriented sub-word sizes $(8,10,12$, and 16 bits) are used in the proposed DCT operator. One dimensional DCT and twodimensional processes were shown by Fig. 4 and Fig. 5 in this paper.

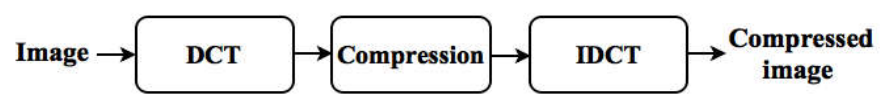

Fig. 4 Image compression process [5].

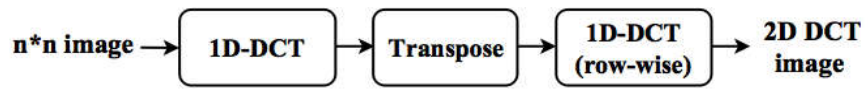

Fig. 5 Two-dimensional DCT operation [5].

\section{Discrete Wavelet transform}

The Fourier transform has been based on image processing. A recent transformation, called the wavelet transform, with the help of this technique it becomes even more easy to analyze and also easier to compress the images. This section describes the DWT in details with the previous research work. For example in [6], inquires the excellence of several wavelet planes and the size of various surroundings on the perfection of image de-noising algorithms concerning PSNR. Because of the capability to capture the energy of a signal in little energy transforms values of wavelet; these techniques are very effective for de-noising of natural images tainted by Gaussian noise. Because of the ability to capture the energy of a signal in little energy transforms values of wavelet; these techniques are very effective for de-noising of natural images corrupted by Gaussian noise. Usual de-noising algorithms which treat the wavelet transform are the formation of three orders. Those are noisy signal is counted in the wavelet transform, according to some rule the noisy wavelet coefficients are modified and using the modified coefficients inverse transform is computed. Processes how DWT works is shown in this paper by Fig. 6 . And decompressed image histogram of DWT decompressed image is shown in Fig. 7.

Because of the many advantages the wavelets, in [2], gave a reason to illustrate a signal in time-frequency and depend over small waves for different dimensions to the compression, called wavelets which are of confined duration and dissimilar frequency. Discrete Wavelet Transform (DWT) uses multiple resolutions. DWT has enticed more interest in image denoising. These experiments are directed to study the suitability of various wavelet bases and window sizes.

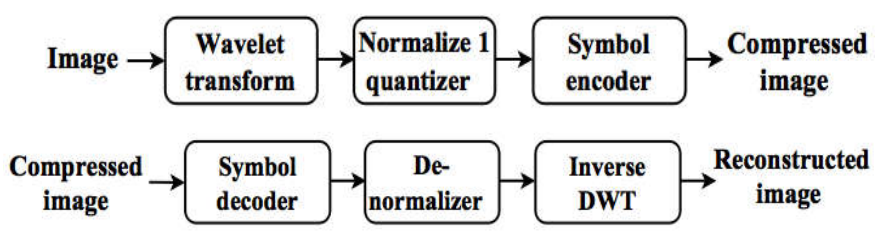

Fig. 6 Image Compression Using Wavelets [2]. 


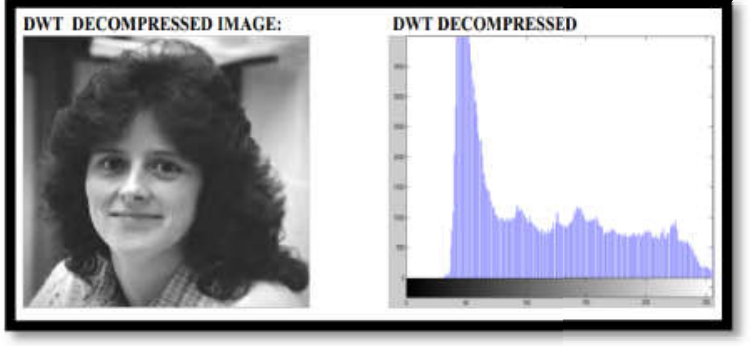

Fig. 7 Image Compression Using Wavelets [2].

\section{Comparison between DCT and DWT}

The basic comparison of DCT, as well as DWT is provided in this section. In [7], performance analysis and comparative study of DWT and DCT image compression algorithm in a wireless sensor network are shown. Besides, the algorithm of DCT and DWT are proposed and comparison between them had shown.

In DCT algorithm, Firstly, the image is changed into an ordination satisfactory for image compression. Then, the image is split into $8 \times 8$ blocks. The coming step is involved in the transformation of the block into the frequency plane to utilize local compatibility among pixels done by using a fast discrete cosine transform (FDCT). The zigzag method is treated to cast the most likely non-zero elements first and the zero elements in the bit-stream. Then entropy coding is used which a summation of variable length coding and Huffman is encoding, and ultimately, a suitable data packet is raised for conduction with the help of the Wireless sensor network.

In DWT algorithm, in an image compression, the image is converted into a compatible format. In the first level, the filters spilled the input image into four non-overlapping multiresolution sub-bands LL, LH, HL, and HH. Thereafter, quantification and coding of sub-bands are used to lessen the number of bits wanted to picture the image. The coming step is arithmetic coding that is a variable length coding which enciphers the original message entirely and illustrates a single number. At last, for transmission with the help of the Wireless sensor network a suitable data packet is raised.

Finally, this paper has observed that MSE is low and PSNR is high in DWT than DCT based compression by considering several images as inputs. From the results, it was terminated that in total performance of DWT is better than DCT on the basis of compression rates.

\section{Polar Coding}

The merits and demerits of the polar coding are described in this section based on the previous research works. Such as in [8], the performance of Polar codes was compared with ReedMuller codes. Advantages of polar codes for performance over reed-Muller are illustrated which are mainly compared between those two. Also, demonstrates that to raise the performance of Reed-Muller (RM) codes the polar coding idea was used. It is done without growing their encoding and decoding complexity.
Polar coding is a construction method of code. It can acquire the capacity of symmetric binary-input discrete memoryless channels. The channels are binary erasure channel (BEC) and binary symmetric channel (BSC). RM codes can be taken into consideration as codes on graphs were showed by Forney, and consequently, decoded by BP decoders illustrated in [8]. By BP decoders polar codes can be decoded too. It is because Polar codes are sub-codes of the full $\operatorname{RM}(n, n)$ codes. This paper considers especially polar codes and BP decoding of RM.

In code construction, one may look for better performance at the consumption of more complexity. It is for polar code construction rules that are tailored to the tangible channel on which the code will be used.

In [9], the performance of Polar Codes is analyzed. This paper also discusses various techniques, by these techniques finite length performance of polar codes is improved. Besides the performance of these codes were studied over source coding. Polar codes are the first family of codes. It is known to achieve a capacity of symmetric channels using a low complexity successive cancellation decoder. These codes are optimal although combined with successive cancellation and their finite length performance is not record breaking.

By studying the performance of these codes over source coding, it is shown that in the unique user and distributed applications polar coding is lossy and lossless. The lossless problem is mapped to the channel coding problem over a binary symmetric channel (BSC). The lossy source coding is a problem of channel coding. However, it represents the process of creating a better source code through the better channel code.

In [10], multidimensional polar encoding and decoding have discussed. Their aim is to update the operation efficiency of the polar codes. And that is done by extending the symbol of polar transformation into multi-dimensional polar codes. Also applying soft decoding and iterative detection and decoding methods. Both the latency and space complexity of the decoder is $O(N)$, where $\mathrm{N}$ is the length of the code specified in [10]. They extended the polar transformation into twodimensional polar transformation and then into multidimensional polar transformation.

Overall, it is concluded from above works is that performance of polar coding is comparatively better. Its advantages can be summarized. That is, it reduces the power required to transmit the signal also DC component problem seen in unipolar encoding is alleviated.

\section{Conventional Encoding}

The common encoding techniques that are used in the various research works are a Run-length encoder, Huffman encoder, Arithmetic encoder. In [11], how arithmetic coding works and efficient implementations is described. Also, the role of probability models and how they provide probability information to the arithmetic coder are discussed. The use of Integer Arithmetic is also described. Integers for the frequency counts used to estimate event probabilities are used. This 
paper also illustrates the gains and weaknesses of arithmetic coding. Its advantages are its optimality and its inherent separation of coding and modeling for statistical data compression. It is easy to maintain lexicographic order without any loss in compression efficiency. The disadvantages of arithmetic coding are that it runs slowly. As well it is fairly difficult to use, and it is not able to provide the prefix codes.

In [12], Huffman algorithm is compared with other common compression techniques like LZW, Run-Length Encoding, and Arithmetic. And it is shown that Huffman coding is comparatively better than other mentioned coding. Huffman coding is a lossless data compression technique. It is based on the frequency of occurrence of a data item i.e. pixel in images. The technique is to use a lower number of bits to encode the data into binary codes that occur more frequently. It is used in JPEG files. Also, a basic code is specified in [12]. Advantages of Huffman coding are that it is easy to implement and a lossless compression of the image is produced. Also, it is very effective in conjunction with a semi-adaptive model [11]. Disadvantages of it are that it is relatively slow, relays upon the statistical model of data, decoding is difficult due to different code lengths, and overhead due to Huffman tree which is cited in [12].

In [13], color image compression is illustrated based on Wavelet Packet Best Tree.This paper suggests improving the run length encoding method which shows better performance than Run-Length Encoding (RLE). Simple RLE has demonstrated very operative encoding in numerous submissions. It is a pattern recognition structure that quests for the reappearance of a like data values in the code-stream. They planned the adjusted technique for the encoding named as Enhanced Run-Length Encoding. This paper also has referenced the problems of surviving Run Length Encoding in [13]. The problem is the compression ratio that is gained from run length encoding systems contrast reliant on the form of data to be encoded, and the reappear acnes existent within the data set. This problem is abolished up to the convinced amount by using Enhanced Run-Length Encoding skill. In the proposed Enhanced RLE, the adjacent coefficients have compared with a standard value, which is afforded by the user according to the applications. Then the changes are undone if the modification is less than the tolerable value. From [12], it is known that gains of RLE are that it is simple to implement and fast to execute. Also, it is a lossless compression. And compression ratio is low as compared to other algorithms is the drawback.

In [14], the convolution encoding with the decoding based on the Viterbi algorithm is illustrated. Bit Error Rate in comparison with the un-coded data analyzes the performance of the code.

The complexity of the decoder increases with the increase in constraint length. BPSK modulation system in symmetric AWGN channel in MATLAB is used to perform the simulation. Forward Error Correction (FEC) which is automatically detected and corrects the errors by adding redundant bits to the message signal and thereby increasing the bandwidth of the signal. The convolution codes are a forward error correction technique deals with the random errors involves the addition of redundant bits which determines the generated error due to the presence of noise in the channel. Convolution Encoder is the length of constraint in the convolution encoder is fixed. Convolution Decoder algorithm is considered and explored by Viterbi in that algorithm highest possibility decipherment is carried out that is judged as an action which reduces the countable load. Bit Error Rate (BER), i.e. Number of error bits/ total transmitted bits determines the performance criterion during the digital data transmission. The signal and noise are explained by Signal-to-noise ratio (SNR).

In [15], analyzed different rates for convolution codes and evaluating performance. Binary symmetric channel (BSC) model symmetric channel (BSC) model and binary symmetric channel (BSC) model are used. Convolution encoder is presented first and then added additive white Gaussian noise (AWGN). Digital wireless communication system to detect and correct errors of the received signal convolution code is commonly used. For decoding process uses Vetribi Algorithm. Using MATLAB with Simulink tools simulations are carried out. Both coded and encoded are compared for bit error rate (BET) is used as testing parameter and results of system behavior.

All the coding techniques have some advantages and disadvantages. To avoid the short comes of those coding, preserving the assets of polar coding.

\section{Hierarchical Quadrature Amplitude Modulation (HQAM)}

To provide the extra security of the high priority data in the transmission medium the HQAM is introduced. The in details explanation of HQAM is provided in this section with advantage and disadvantage. In [16], UEP of data transmission treating HQAM and channel coding are inquired. To offer various degrees of error saving of the highest significant report of data content the M-QAM constellations are used in that the isolated data is sent through two different M-QAM transmissions over an AWGN channel. The MSBs and LSPs respectively are carried by the two sub-channels HP and LP and the performance is measured by Additive Gaussian Noise (AWGN). By changing the alpha value 16-QAM is also proposed. To achieve a more robust SNR channel coding is used. More protection is given to the HP channel that changing the value of alpha at the charge of the LP channel for coded and non-coded channels which is observed from the results which generates a strong quality of the reformed data likened with the equal guard.

In [17], inquired the performance of HQAM. In HQAM nonuniform signal constellation is utilized which provides various degrees of protection in the compressed image data at lower channel SNR. And that is compared with QAM. Higher transmission efficiency is provided by QAM by improving both phase and amplitude variations. And it provides more protection for higher priority bits of the image data by HQAM. HQAM is the upgrade version of QAM, which is used in the image transmission over the noisy channel. Different values of the modulation parameters are applied on the gray test image for evaluating the performance of HQAM. Below Fig. 8 shows the steps of simulation which is offered in this paper. 


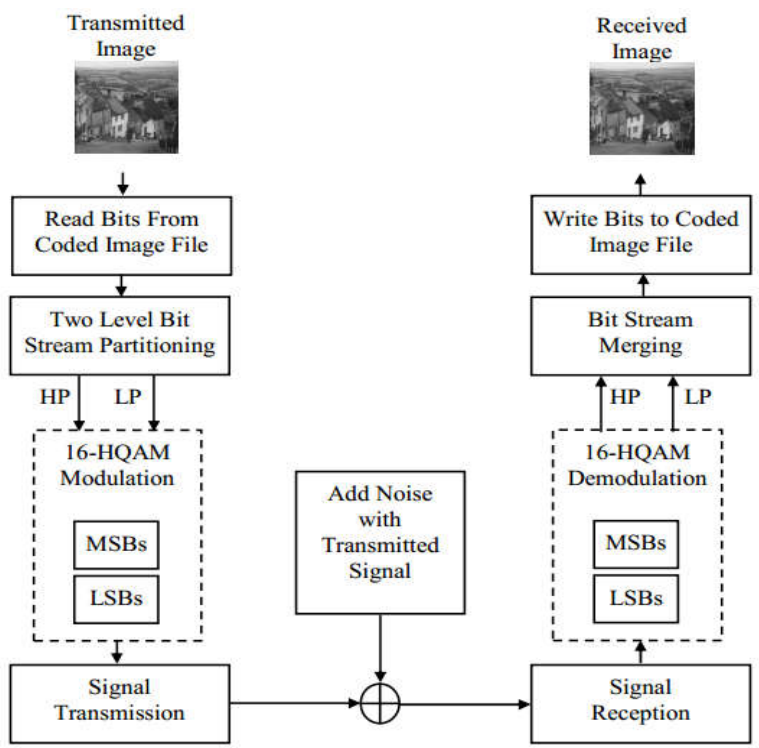

Fig. 8 Working procedure of the image transmission using 16-HQAM [18].

In [18], image transmission suitability of using Hierarchical 16-QAM as modulation technique to give several degrees of protection non-uniform signal constellation is used for the non-significant and significant bit sand as an image coder SPIHT (Set Partitioning in Hierarchical Trees). Evaluated performance of SPIHT is coded image transmission for different values of the modulation parameter in Simulink using gray test image and bit per pixel (bpp). Simulink model shows for image communication through erroneous wireless channels suitability of using it from the simulation results and verifies the theory of HQAM.

In [19], proposed a higher order QAM therein non-uniform signal constellation including hierarchically oriented OFDM notation to give greater benefit to reduced image information at a minor way of SNR and lesser BER. HQAM with the help of irregularly spaced constellations and a modification of QAM qualify unbalanced precedence shipment with the help of carelessly placed constellations and an alternation of QAM, grants Unequal Error Protection (UEP) to translated streams for growing the shield to the translated streams and also proficient in power and bandwidth. To explore the perfection of mentioned technique information of theoretical access is applied. How much the mentioned technique outperforms using the approach, it is quantitatively evaluated. Over AWGN environments analytical BER and PSNR of reformatted image of HQAM formats that involved uniform and non-uniform constellations, have been tested. The perfection of mentioned technique and subsisting technique has been accessed. In different wireless reception conditions, hierarchical modulations are initially mentioned to give various classes of data to users.

This skill settles the matter that arrives because of variable bit rate ratio and set located strength for low precedence (LP) and high precedence (HP) data seam by initiating 256 HQAM treatment with OFDM modulator. This system increases the PSNR of received image but lowers down the BER and an efficient means of sending the image via erroneous wireless channel a major modification technique of QAM provides without any additional hardware.

\section{Comparison between HQAM and QAM}

This section provides the comparison of the HQAM and QAM with merits and demerits based on the previous research works. Such as in [20], the compatibility of Hierarchical QAM (HQAM) where non-uniform signal constellation is mannered to give several degrees of security to the significant and nonsignificant bits in the compressed image data. Unequal priority transmission with the help of order less placed constellations is qualified by Hierarchical quadrature amplitude modulation (HQAM) raising the security to the shifted bits and also proficient in bandwidth and strength. HQAM is an easy and proficient way in which a non-uniform signal space constellation is treated to give various units of safeguard. The convenience of this mode is that different grades of shield are acquired but an enhancement in bandwidth; in contradict to channel coding which enhances the data rate by assembling dismissal. About several image transmission skills for acquiring an improved quality and improved speed in digital image transmission in a band-limited fading channel are surveyed in this paper.

Quadrature amplitude modulation (QAM) is a digital modulation procedure in which both the amplitude and phase of a carrier are modulated to transport digital information is also specified in[20].It affords equal error protection to the transmitted bits by assigning identical precedence to both the significant and non-significant bits of data and is categorized as equal error protection (EEP) method of modulation in[2].It is also said in [2] that, Unequal error protection (UEP) is not desirable in QAM is the disadvantage of it. It is understood from the comparison that HQAM is better than QAM in the case of giving protection to the bits.

\section{Performance Analysis of Additive Gaussian Noise (AWGN) Channel}

As AWGN noise has a zero means is variance and powers are identical. In the practical situations, no channel is noise free and all transmitted signal is corrupted by noise. The noise is unpredictable in nature. With consider these situations; this section explains the performance of AWGN with the previous works. Such as in[21], the accomplishment of broadcast styles is accessed by counting the prospect of Bit Error Rate (BER) against the Signal Noise Ratio (SNR) beneath the recurrently mannered three wireless channel models (AWGN, Rayleigh, and Rician). To analyze the performance that is BER vs. SNR, the data modulation, and the data rate is considered and multipath received signals are also considered. The completion of conduction styles within several channel models and a number of antennas from the simulation results. The appreciation of accomplishment authorizes the rise in the coverage area of the physical layer in the 802.11b WLAN devices. Their BER is observed, that is a function of SNR at the recipient, and the performance of data shipment through wireless channels is perfectly taken. Different models have been mentioned and researched to compute SNR in wireless channels.

In [22], performance is evaluated in AWGN Channel for 802.11a High-Speed Network In 802.11a standard of 
multicarrier transmission a single data stream is transmitted over a number of lower rates subcarriers and high data rates provided. A specification for distributing the that are spaced apart at strict frequencies data over a large number of carriers is provided by $802.11 \mathrm{a}$ wireless standard. Orthogonality is provided by this spacing, which obstructs the demodulator from observing frequencies other than their own. Different modulation techniques like as QAM, QPSK, 8PSK, 16PSK, BPSK using MATLAB simulation of the wireless communication system was done is included by $802.11 \mathrm{a}$ wireless standard design. The BPSK allows improving the SNR and BER in a noisy channel at the cost of maximum data transmission capacity. And this is observed from the simulation results. Different modulation techniques are investigated that higher transmission capacity is allowed but at the time of little increase in the probability of error. From the results, the use of 802.11a wireless standard for short distance transmission link with QPSK is favorable. In contrast, BPSK will be preferable for long distance transmission link OFDM.

In [23], tested that for raising the value of data rate will be enhanced but rising value of BER but M-array will act less than QPSK and QAM. For wireless communication measure schemes, QPSK and QAM are considered. Sending of the signal from ground position to moving position treating $\mathrm{M}$ array Quadrature Amplitude modulation (QAM) modulation scheme and Quadrature Phase Shift Keying (QPSK) are consider in WCDMA system. Two measure techniques are explored, 16-QAM then QPSK to minimize the perfection of error in the signal and also assimilate which skill is excellent via AWGN Channel. The performance of W-CDMA method in AWGN channel displays that 16-QAM has a minor function likened to the QPSK modulation technique.

In [24], characterization and the design of analog signal waveforms are focused that carries digital information. Also, performance is compared on an AWGN channel in this paper. For MATLAB simulation purposes a number of modulations BPSK, QPSK, BFSK, DBPSK, M-PSK, M-FSK, and QAM have been considered. For additive white Gaussian noise channel their BER has been evaluated using MATLAB Monte Carlo simulation tool. BPSK is the most effective modulation schemes in a practical communication system.

\section{Proposed Method}

By revising preceding works on the image transmission through the noisy channel it is identified that the main limitations for the transmitting quality image are demanded more storage capacity and higher bandwidth requirement. For that reason, the image compression is performed by utilizing DCT and DWT in the proposed method as these methods have significant advantages. The workflow of the proposed method is shown in Fig. 9. One of the advantages of DCT is that it has the ability to pack energy in the lower frequencies for image data. Whereas, the advantage of DWT is that it uses multiple resolutions besides the wavelets gives away to illustrate a signal in time-frequency.

Another challenge of the transmitting image through the noisy channel is that of encoding the compressed quantized values. The common encoding techniques that are used in the various research works are run-length encoder, Huffman encoder, Arithmetic encoder.

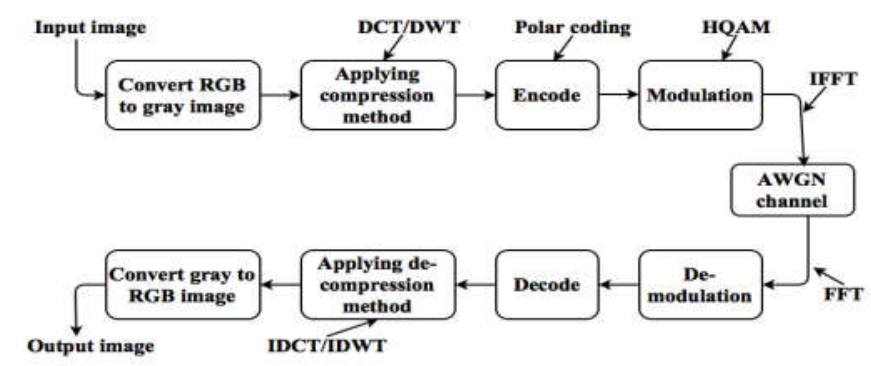

Fig. 9 Workflow of Proposed Method.

However, those encoders have some limitations. One limitation of the Huffman coding is that the binary strings streams in the encoded data are all different lengths. In Runlength-coding, minimum useful run-length size is increased from three characters to four which could affect compression efficiency with some types of data. On the other hand, one of the limitations of Arithmetic coding is that the whole codeword must be received to start decoding the symbols, and if there is a corrupt bit in the codeword, the entire message could become corrupt. Considering all the limitations of the different encoding methods the Polar coding technique is used in the proposed method. It is an efficient encoding and decoding algorithm which lessens the power required to transmit the signal by one-half and it also achieves the capacity.

From the various research works, it is observed that transmitting data bits get less protection. To provide the better protection to the transmit bits from noisy and get limited errorfree data stream, the HQAM modulation technique is introduced in the proposed method. This modulation technique provides unequal error protection (UEP) whereas QAM provides equal error protection (EEP) which was used in many previous works. Furthermore, QAM is more susceptible to noise. Another benefit of HQAM is that unlike degrees of security are attained without increasing the bandwidth.

As the DCT or DWT coefficients are in the frequency domain. However, AWGN model works in the time domain. For that purpose, inverse first Fourier transform (IFFT) is introduced in this proposed method. In the proposed method, the performance of HQAM will be observed in terms of PSNR and MSE both for DCT and DWT gradually. Then both result will be compared and the preferred result will be witnessed.

\section{Conclusion}

To establish an efficient communication model different compression, encoding and modulation techniques have been reviewed in this paper. By reviewing these techniques a communication model is proposed for future work. In this model, two effective compression methods are introduced i.e., DCT and DWT. For better encoding of the compressed date compare with other existing encoding methods, polar coding is selected. In the communication model, it is hard to give better protection to the transmit image date. For that reason, HQAM modulation method is proposed which gives comparatively 
more protection to data and image quality while transmitting image date through the noisy AWGN channel. Finally, the performance of HQAM is analyzed for DWT and DCT after transmitting the image through the noisy AWGN channel.

\section{References}

[1] Y. Soon, S. N. Koh, and C. K. Yeo, "Noisy speech enhancement using discrete cosine transform," Speech communication, Vol. 24, pp. 249257, 1998.

[2] P. Telagarapu, V. J. Naveen, A. L. Prasanthi, and G. V. Santhi, "Image compression using DCT and wavelet transformations," International Journal of Signal Processing, Image Processing and Pattern Recognition, Vol. 4, pp. 61-74, 2011.

[3] A. Raid, W. Khedr, M. El-dosuky, and W. Ahmed, "Jpeg Image Compression Using Discrete Cosine Transform-A Survey," arXiv preprint arXiv:1405.6147, Vol. 5, pp. 47, 2014.

[4] S. Khan, E. Casseau, and D. Menard, "High Performance Discrete Cosine Transform Operator Using Multimedia Oriented Subword Parallelism," Advances in Computer Engineering, 2015.

[5] S. Khan, E. Casseau, and D. Menard, "High Performance Discrete Cosine Transform Operator Using Multimedia Oriented Subword Parallelism," Advances in Computer Engineering, 2015.

[6] S. K. Mohideen, S. A. Perumal, and M. M. Sathik, "Image de-noising using discrete wavelet transform," International Journal of Computer Science and Network Security, Vol. 8, pp. 213-216, 2008.

[7] O. Ghorbel, W. Ayedi, M. W. Jmal, and M. Abid, "Dct \& Dwt Images Compression Algorithms in Wireless Sensors Networks: Comparative Study And Performance Analysis," International Journal of Wireless \& Mobile Networks, Vol. 4, p. 45, 2012.

[8] E. Arıkan, "A performance comparison of polar codes and Reed-Muller codes," IEEE Commun. Lett, Vol. 12, pp. 447-449, 2008.

[9] N. Hussami, S. B. Korada, and R. Urbanke, "Performance of polar codes for channel and source coding," in 2009 IEEE International Symposium on Information Theory, pp. 1488-1492, 2009.

[10] H. Mahdavifar, M. El-Khamy, J. Lee, and I. Kang, "Fast multidimensional polar encoding and decoding," in Information Theory and Applications Workshop (ITA), pp. 1-5, 2014.

[11] I. H. Witten, R. M. Neal, and J. G. Cleary, "Arithmetic coding for data compression," Communications of the ACM, vol. 30, pp. 520-540, 1987.
[12] M. Sharma, "Compression using Huffman coding," IJCSNS International Journal of Computer Science and Network Security, Vol. 10, pp. 133-141, 2010.

[13] G. K. Kharate and V. H. Patil, "Color image compression based on Wavelet packet Best Tree," arXiv preprint arXiv:1004.3276, 2010.

[14] M. Pandey and V. K. Pandey, "Implementation and Performance Analysis of Convolutional Encoder and Viterbi Decoder Using Matlab."

[15] M. Hamdan and A. Abdullah, "Analysis and Performance Evaluation of Convolutional Codes over Binary Symmetric Channel Using MATLAB".

[16] A. B. Abdurrhman and M. E. Woodward, "Unequal error protection for data transmission using adaptive hierarchical QAM," in 9th Annual Postgraduate Symposium on The Convergence of Telecommunications, Networking and Broadcasting, pp. 1153-1158, 2008.

[17] F. G. Md. Abdul Kader, and R. Badlishah Ahmad, "Design and Development of Compressed Image Transmission System Using Asymmetric Modulation (HQAM) Over Wireless Channels," Int. Conf. on Advances in Computing, Control, and Telecommunication Technologies, pp. 131-135, 2011.

[18] M. A. Kader, F. Ghani, and R. Badlishah, "Development and performance evaluation of hierarchical quadrature amplitude modulation (HQAM) for image transmission over wireless channels," in Computational Intelligence, Modelling and Simulation (CIMSiM), 2011 Third International Conference on, pp. 227-232, 2011.

[19] C. B. Preeti Rani, "MIMO-OFDM Communication System Design using 256 HQAM," International Journal of Advanced Research in Computer Science and Software Engineering, Vol. 4, pp. 558-563, 2014

[20] N. Sharma, "Designing and Comparative Analysis of Image Transmission using Techniques HQAM and QAM."

[21] A. S. Babu and D. K. S. Rao, "Evaluation of BER for AWGN, Rayleigh and Rician fading channels under various modulation schemes," International Journal of Computer Applications, Vol. 26, 2011.

[22] D. M. A. 1, A. Kumar, "Performance Evolutionary in AWGN Channel for 802.11a High Speed Network," International Journal of Advanced Research in Computer Science and Software Engineering, Vol. 2, pp. 5, 2012.

[23] M. S. I. M. H. R. J. Sultana, "Performance Analysis of QAM and QPSK in AWGN and Multipath Fading Channel," International Journal of Advanced Research in Computer Science and Software Engineering, Vol. 5, pp. 1648-1654, 2015.

[24] G. Sadeque, "Bit Error Rate (BER) Comparison of AWGN Channels for Different Type's Digital Modulation Using MATLAB Simulink," American Scientific Research Journal for Engineering, Technology, and Sciences (ASRJETS), Vol. 13, pp. 61-71, 2015. 